\title{
General computational approach for optimal fault detection
}

\author{
Andras Varga \\ German Aerospace Center, DLR-Oberpfaffenhofen \\ Institute of Robotics and Mechatronics \\ D-82234 Wessling, Germany \\ Email: andras.varga@dlr.de
}

\begin{abstract}
We propose a new computational approach to solve the optimal fault detection problem in the most general setting. The proposed procedure is free of any technical assumptions and is applicable to both proper and non-proper systems. This procedure forms the basis of an integrated numerically reliable state-space algorithm, which relies on powerful descriptor systems techniques to solve the underlying computational subproblems. The new algorithm has been implemented into a Fault Detection Toolbox for MATLAB.
\end{abstract}

Keywords: Fault detection, H2/Hinf-optimal synthesis, computational methods.

\section{INTRODUCTION}

The fault detection problem consists in detecting via socalled residual signals (or residuals) the occurrence of any fault in a system in the presence of arbitrary control and disturbance inputs acting on that system. The residuals are generated by a residual generator filter having as inputs the measured outputs and the controlled inputs. For the exact or approximate solution of fault detection problems we need to synthesize residual generator filters which are highly sensitive to all faults in the presence of controls and disturbances acting on the system. This problem has been widely studied using different problem settings and different solution approaches. For a comprehensive account of existing methods see for example the monographs of Gertler [1998], Chen and Patton [1999] as well as some of recent publications [Ding et al., 2000, Jaimoukha et al., 2006, Liu and Zhou, 2007a,b, Zhang and Ding, 2008].

The conditions for the exact solvability of the fault detection problem are frequently not fulfilled in practical applications. This is usually the case, for example, when robustness aspects are addressed by recasting uncertain parameters as additional (artificial) disturbance inputs. Thus, in most of applications only approximate solutions of the fault detection problem can be aimed to be computed, where the goal is to design residual generators which minimize the effects of disturbances on the residuals, while simultaneously maximizing the effect of faults. The approximate solution of the fault detection problem has been addressed by many authors [Ding et al., 2000, Jaimoukha et al., 2006, Liu and Zhou, 2007a,b, Zhang and Ding, 2008] by solving various multi-objective optimization problems, as for example, the $\mathcal{H}_{-} / \mathcal{H}_{\infty}$ and $\mathcal{H}_{-} / \mathcal{H}_{2}$ optimal fault detection problems. A common feature of some of the proposed solution approaches is that they usually rely on various technical assumptions which,

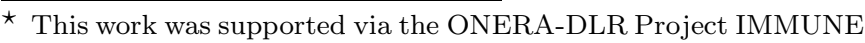
Intelligent Monitoring and Management of Unexpected Events. although allows the derivation of explicit analytical solutions, are not really necessary for the solution of the problem. Moreover, often the connections between the exact and approximate solutions are obscured either by employing inappropriate optimization criteria or completely ignoring available structural information. This has a rather undesirable consequence that the exact solution can not be computed via the proposed solution methods even if it exists (see the example employed in Jaimoukha et al. [2006] and Example 2. of [Liu and Zhou, 2007a].)

In this paper, we propose an enhanced computational approach to solve the fault detection problem in the most general setting. The proposed procedure is free of any technical assumptions and is applicable to both proper as well as non-proper systems. This procedure forms the basis of a numerically reliable state-space computational algorithm. This algorithm relies on powerful descriptor systems computational techniques to solve the underlying computational subproblems. A main feature of the proposed computational approach is that the successive solution steps are fully integrated in an overall synthesis procedure by employing appropriate updating techniques. The proposed new synthesis procedure has been implemented as robust numerical software which is now part of the Fault Detection Toolbox for Matlab developed by the author [Varga, 2006].

\section{THE FAULT DETECTION PROBLEM}

Consider additive fault models described by input-output representations of the form

$\mathbf{y}(\lambda)=G_{u}(\lambda) \mathbf{u}(\lambda)+G_{d}(\lambda) \mathbf{d}(\lambda)+G_{w}(\lambda) \mathbf{w}(\lambda)+G_{f}(\lambda) \mathbf{f}(\lambda)$,

where $\mathbf{y}(\lambda), \mathbf{u}(\lambda), \mathbf{d}(\lambda), \mathbf{w}(\lambda)$, and $\mathbf{f}(\lambda)$ are Laplace- or Z-transformed vectors of the $p$-dimensional system output vector $y(t), m_{u}$-dimensional control input vector $u(t), m_{d^{-}}$ dimensional disturbance vector $d(t), m_{w}$-dimensional noise vector $w(t)$ and $m_{f}$-dimensional fault vector $f(t)$, respectively, and where $G_{u}(\lambda), G_{d}(\lambda), G_{w}(\lambda)$ and $G_{f}(\lambda)$ are the 
transfer-function matrices (TFMs) from the control inputs to outputs, disturbance inputs to outputs, noise inputs to outputs, and fault inputs to outputs, respectively. According to the system type, the frequency variable $\lambda$ is either $s$, the complex variable in the Laplace-transform in the case of a continuous-time system or $z$, the complex variable in the Z-transform in the case of a discrete-time system. For most of practical applications, the TFMs $G_{u}(\lambda), G_{d}(\lambda)$, $G_{w}(\lambda)$ and $G_{f}(\lambda)$ are proper rational matrices. However, for complete generality of our problem setting, we will allow that these TFMs are general non-proper rational matrices for which we will not a priori assume any further properties (e.g., stability, full rank).

A linear residual generator (or fault detection filter) processes the measurable system outputs $y(t)$ and control inputs $u(t)$ and generates the residual signals $r(t)$ which serve for decision making on the presence or absence of faults. The input-output form of this filter (also called the computational form by Gertler [1998]) is

$$
\mathbf{r}(\lambda)=R(\lambda)\left[\begin{array}{l}
\mathbf{y}(\lambda) \\
\mathbf{u}(\lambda)
\end{array}\right]
$$

where $R(\lambda)$ is the TFM of the filter. For a physically realizable filter, $R(\lambda)$ must be proper (i.e., only with finite poles) and stable (i.e., only with poles having negative real parts for a continuous-time system or magnitudes less than one for a discrete-time system). The (dynamic) order of $R(\lambda)$ (also known as McMillan degree) is the dimension of the state vector of a minimal state-space realization of $R(\lambda)$. The dimension $q$ of the residual vector $r(t)$ depends on the fault detection problem to be solved, and can be either given or determined during the solution process.

The residual signal $r(t)$ in (2) generally depends via the system outputs $y(t)$ of all system inputs $u(t), d(t), w(t)$ and $f(t)$. The residual generation system (also called the internal form of residual generator by Gertler [1998]) is obtained by replacing in $(2) \mathbf{y}(\lambda)$ by its expression in (1) $r(\lambda)=R_{u}(\lambda) \mathbf{u}(\lambda)+R_{d}(\lambda) \mathbf{d}(\lambda)+R_{w}(\lambda) \mathbf{w}(\lambda)+R_{f}(\lambda) \mathbf{f}(\lambda)$

where

$$
\begin{aligned}
& {\left[R_{u}(\lambda)\left|R_{d}(\lambda)\right| R_{w}(\lambda) \mid R_{f}(\lambda)\right]:=} \\
& R(\lambda)\left[\begin{array}{c|c|c|c}
G_{u}(\lambda) & G_{d}(\lambda) & G_{w}(\lambda) & G_{f}(\lambda) \\
I_{m_{u}} & 0 & 0 & 0
\end{array}\right]
\end{aligned}
$$

For a successfully designed filter $R(\lambda)$, the corresponding residual generation system is proper and stable and achieves specific fault detection requirements (e.g., decoupling of control and disturbance inputs from the residuals).

In this paper we consider the solution of the following approximate fault detection problem (AFDP): For given $\gamma>0$, determine $\beta>0$ and a physically realizable linear residual generator filter of the form (2) (with possibly least order) such that for all $u(t)$ and $d(t)$ we have:

(i) $\|r(t)\| \leq \gamma\|w(t)\|$ when $f(t)=0$ and for all $w(t)$;

(ii) $\|r(t)\| \geq \beta\left|f_{j}(t)\right|$ for $j=1, \ldots, m_{f}$ and for all $w(t)$.

This formulation of AFDP includes the exact fault detection problem (EFDP) when $m_{w}=0$, as well as the alternative formulations in [Ding et al., 2000, Liu and Zhou, 2007a,b] when $m_{d}=0$. Moreover, this formulation of the AFDP also covers structured residuals, where part of the disturbance signals in $d(t)$ are faults which must be decoupled from the residuals Gertler [1998].

For the solution of the AFDP, the solvability conditions are the well known conditions for the EFDP (see for example [Saberi et al., 2000, Theorem 2.2]):

Theorem 1. For the system (1) the AFDP is solvable if and only if

$\operatorname{rank}\left[G_{d}(\lambda) G_{f_{j}}(\lambda)\right]>\operatorname{rank} G_{d}(\lambda), \quad j=1, \ldots, m_{f}$ where $G_{f_{j}}(\lambda)$ is the $j$-th column of $G_{f}(\lambda)$.

For the case $m_{d}=0$ considered in [Ding et al., 2000, Liu and Zhou, 2007a,b], the above conditions reduce to the output observability conditions:

$$
G_{f_{j}}(\lambda) \neq 0, \quad j=1, \ldots, m_{f}
$$

The solution of the AFDP can be addressed by reformulating it into an optimization problem with constraints. To measure the sensitivity of residuals to faults, we use the following $\mathcal{H}_{-}$index

$$
\left\|R_{f}(\lambda)\right\|_{-}=\min _{j}\left\|R_{f_{j}}(\lambda)\right\|_{\alpha}
$$

where $\alpha=2$ or $\infty$. The requirement $\left\|R_{f}(\lambda)\right\|_{-}>0$ merely asks for nonzero columns $R_{f_{j}}(\lambda)$ and thus is equivalent to a (weak) fault detectability condition. Note that the alternative $\mathcal{H}_{-}$indices used in [Ding et al., 2000, Jaimoukha et al., 2006, Liu and Zhou, 2007a,b, Zhang and Ding, 2008] are defined in terms of the least singular values of $R_{f}(\lambda)$ and thus are applicable only when $m_{f} \leq p$.

With the definition (6) of the $\mathcal{H}_{-}$index, we can solve the AFDP as an $\mathcal{H}_{-} / \mathcal{H}_{\infty}$ or $\mathcal{H}_{-} / \mathcal{H}_{2}$ optimization problem:

AFDPO - For a given $\gamma>0$, determine a proper and stable $R(\lambda)$ such that $R_{u}(\lambda)=0, R_{d}(\lambda)=0, R_{f}(\lambda)$ and $R_{w}(\lambda)$ are proper and stable, and $R(\lambda)$ solves

$$
\beta:=\max \left\{\left\|R_{f}(\lambda)\right\|_{-}:\left\|R_{w}(\lambda)\right\|_{\alpha} \leq \gamma\right\}>0
$$

The achieved gap $\beta / \gamma$ can be interpreted as a performance measure of the noise rejection properties of the detector.

In this paper we propose a general approach to solve the AFDP. The AFDPO Procedure given bellow is a combination of the nullspace approach of [Varga, 2003] to design least order fault detection filters to solve the EFDP with an optimization based solution inspired by the methods proposed in [Ding et al., 2000, Liu and Zhou, 2007a,b] to solve the AFDP with $m_{d}=0$. In this procedure, a desired dimension of the residual vector $q$ can be specified, but it is updated if necessary during the solution procedure. The value $q=1$ can be always employed whenever a solution exists and leads to a least order detector. However, larger values of $q$ provide additional freedom to increase the gap $\beta / \gamma$. The computational ingredients of the procedure are discussed in the next section.

\section{AFDPO Procedure.}

Step 1. Choose $R(\lambda)$ of the form

$$
R(\lambda)=Q(\lambda) R_{1}(\lambda),
$$

where $R_{1}(\lambda)$ is a $q_{1} \times\left(p+m_{u}\right)$ TFM which solves the EFDP assuming $w(t) \equiv 0$. Compute

$$
\widetilde{G}_{f}(\lambda)=R_{1}(\lambda)\left[\begin{array}{c}
G_{f}(\lambda) \\
0
\end{array}\right], \quad \widetilde{G}_{w}(\lambda)=R_{1}(\lambda)\left[\begin{array}{c}
G_{w}(\lambda) \\
0
\end{array}\right]
$$

and determine $r_{w}:=\operatorname{rank} \widetilde{G}_{w}(\lambda)$. 
If $r_{w}=0$, choose $q_{1}=\min \left(p-r_{d}, q\right)$, where $r_{d}=$ $\operatorname{rank} G_{d}(\lambda)$ and $p-r_{d}$ is the dimension of the left nullspace of $G_{d}(\lambda)$. Set $q=q_{1}, N_{f}(\lambda)=\widetilde{G}_{f}(\lambda), R_{2}(\lambda)=R_{1}(\lambda)$, and go to Step 4; else, choose $q_{1}=r_{w}$. The optimization problem (7) becomes in this case to determine a $q \times q_{1}$ proper and stable TFM $Q(\lambda)$ which solves

$$
\beta=\max \left\{\left\|Q(\lambda) \widetilde{G}_{f}(\lambda)\right\|_{-}:\left\|Q(\lambda) \widetilde{G}_{w}(\lambda)\right\|_{\alpha} \leq \gamma\right\}>0
$$

Step 2. Compute the quasi-coouter-coinner factorization of $\widetilde{G}_{w}(\lambda)$ in the form

$$
\widetilde{G}_{w}(\lambda)=G_{w o}(\lambda) G_{w i}(\lambda),
$$

where the quasi-coouter factor $G_{w o}(\lambda)$ is a $q_{1} \times q_{1}$ invertible TFM which has only stable zeros, excepting possible zeros on the imaginary axis (including infinity) for a continuoustime system or on the unit circle for a discrete-time system, and $G_{w i}(\lambda)$ is coinner (i.e., $\left.G_{w i}(\lambda) G_{w i}^{*}(\lambda)=I_{q_{1}}\right)$. The poles of $G_{w o}(\lambda)$ are those of $\widetilde{G}_{w}(\lambda)$.

The optimization problem (8) can be equivalently reformulated as

$$
\beta=\max \left\{\left\|Q(\lambda) \widetilde{G}_{f}(\lambda)\right\|_{-}:\left\|Q(\lambda) G_{w o}(\lambda)\right\|_{\alpha} \leq \gamma\right\}>0
$$

Step 3. Compute $Q(\lambda)$ of the form

$$
Q(\lambda)=\Theta(\lambda) G_{w o}^{-1}(\lambda)
$$

If we denote

$$
N_{f}(\lambda)=G_{w o}^{-1}(\lambda) \widetilde{G}_{f}(\lambda),
$$

then the optimization problem $(9)$ reduces to choose the optimal $\Theta(\lambda)$ which solves

$$
\beta=\max \left\{\left\|\Theta(\lambda) N_{f}(\lambda)\right\|_{-}:\|\Theta(\lambda)\|_{\alpha} \leq \gamma\right\}>0
$$

The updated detector has the form

$$
R_{2}(\lambda)=G_{w o}^{-1}(\lambda) R_{1}(\lambda)
$$

Step 4. Compute the proper and stable factorization

$$
\left[R_{2}(\lambda) N_{f}(\lambda)\right]=M_{w}^{-1}(\lambda)\left[R_{3}(\lambda) \tilde{N}_{f}(\lambda)\right]
$$

where $M_{w}(\lambda), R_{3}(\lambda)$ and $\tilde{N}_{f}(\lambda)$ are proper and stable TFMs. If $r_{w}=0$, set $R(\lambda)=R_{3}(\lambda), R_{f}(\lambda)=\tilde{N}_{f}(\lambda)$, $R_{w}(\lambda)=0$, and Exit; else, with $\Theta(\lambda)=\Psi(\lambda) M_{w}(\lambda)$, the optimization problem (10) becomes to choose the optimal $\Psi(\lambda)$ which solves

$$
\beta=\max \left\{\left\|\Psi(\lambda) \tilde{N}_{f}(\lambda)\right\|_{-}:\left\|\Psi(\lambda) M_{w}(\lambda)\right\|_{\alpha} \leq \gamma\right\}>0
$$

Step 5. Choose $\Psi(\lambda)$ a $q \times q_{1}$ TFM such that

and

$$
\left\|\Psi(\lambda) M_{w}(\lambda)\right\|_{\alpha}=\gamma
$$

$$
\beta=\left\|\Psi(\lambda) \tilde{N}_{f}(\lambda)\right\|_{-}>0
$$

Set $R(\lambda)=\Psi(\lambda) R_{3}(\lambda), \quad R_{f}(\lambda)=\Psi(\lambda) \tilde{N}_{f}(\lambda)$, and $R_{w}(\lambda)=\Psi(\lambda) M_{w}(\lambda) G_{w i}(\lambda)$.

In what follows, we describe a state space computations based version of this algorithm. For each step, we discuss the necessary computational ingredients using descriptor system representations. Explicit state space realizations are derived for the TFMs $R_{3}(\lambda), \tilde{N}_{f}(\lambda)$ and $M_{w}(\lambda)$.

\section{COMPUTATIONAL ISSUES}

For computations we exclusively rely on an equivalent descriptor state space realization of the input-output model (1),

$$
E \lambda x(t)=A x(t)+B_{u} u(t)+B_{d} d(t)+B_{w} w(t)+B_{f} f(t)
$$$$
y(t)=C x(t)+D_{u} u(t)+D_{d} d(t)+D_{w} w(t)+D_{f} f(t)
$$

with the $n$-dimensional state vector $x(t)$, where $\lambda x(t)=$ $\dot{x}(t)$ or $\lambda x(t)=x(t+1)$ depending on the type of the system, continuous or discrete, respectively. In general, the square matrix $E$ can be singular, but we will assume that the linear pencil $A-\lambda E$ is regular. For systems with proper TFMs in (1), we can always choose a standard state space realization where $E=I$. In general, we can assume that the representation (13) is minimal, that is, the descriptor pair $(A-\lambda E, C)$ is observable and the pair $(A-$ $\left.\lambda E,\left[\begin{array}{llll}B_{u} & B_{d} & B_{w} & B_{f}\end{array}\right]\right)$ is controllable. The corresponding TFMs of the model in (1) are

$$
\begin{aligned}
& G_{u}(\lambda)=C(\lambda E-A)^{-1} B_{u}+D_{u} \\
& G_{d}(\lambda)=C(\lambda E-A)^{-1} B_{d}+D_{d} \\
& G_{w}(\lambda)=C(\lambda E-A)^{-1} B_{w}+D_{w} \\
& G_{f}(\lambda)=C(\lambda E-A)^{-1} B_{f}+D_{f}
\end{aligned}
$$

or in an equivalent notation

$\left[G_{u}(\lambda) G_{d}(\lambda) G_{w}(\lambda) G_{f}(\lambda)\right]:=\left[\begin{array}{c|cccc}A-\lambda E & B_{u} & B_{d} & B_{w} & B_{f} \\ \hline C & D_{u} & D_{d} & D_{w} & D_{f}\end{array}\right]$

Step 1. If we employ recently developed synthesis algorithms based on rational nullspace methods [Varga, 2008], we obtain the preliminary filter $R_{1}(\lambda)$ and the corresponding $\widetilde{G}_{f}(\lambda)$ and $\widetilde{G}_{w}(\lambda)$ with realizations of the form

$$
\left[R_{1}(\lambda): \widetilde{G}_{f}(\lambda) \widetilde{G}_{w}(\lambda)\right]=\left[\begin{array}{c|c|c:cc}
\widetilde{A}-\lambda \widetilde{E} & \widetilde{B}_{y} & \widetilde{B}_{u} & \widetilde{B}_{f} & \widetilde{B}_{w} \\
\hline \widetilde{C} & \widetilde{D}_{y} & \widetilde{D}_{u} & \widetilde{D}_{f} & \widetilde{D}_{w}
\end{array}\right]
$$

where $\widetilde{E}$ is invertible (thus all TFMs are proper) and the pair $(\widetilde{A}, \widetilde{E})$ has only finite generalized eigenvalues which can be placed arbitrarily. Moreover, for any given $q \leq p-$ $r_{d}$, a least order detector $R_{1}(\lambda)$ with $q_{1}$ outputs $\left(q_{1} \leq q\right)$ can be determined [Varga, 2003, 2008].

Step 2. For the computation of the quasi-coouter-coinner factorization of $\widetilde{G}_{w}(\lambda)$ we employ the dual of the algorithm of Oară and Varga [2000] for the continuous-time case and the dual of the algorithm of Oară and Varga [1999] for the discrete-time case. In both cases, the quasi-coouter factor $G_{w o}(\lambda)$ is obtained in the form

$$
G_{w o}(\lambda)=\left[\begin{array}{c|c}
\widetilde{A}-\lambda \widetilde{E} & \bar{B}_{w} \\
\hline \widetilde{C} & \bar{D}_{w}
\end{array}\right]
$$

where $\bar{B}_{w}$ and $\bar{D}_{w}$ are matrices with $r_{w}$ columns, $r_{w}$ being the rank of $\widetilde{G}_{w}(\lambda)$. Recall that if $r_{w}>0$, we take $q_{1}=r_{w}$, thus $G_{w o}(\lambda)$ is square. The system with the TFM $G_{w o}(\lambda)$ contains all zeros of $\widetilde{G}_{w}(\lambda)$ on the imaginary axis (including infinity) in the continuous-time case or on the unit circle in the discrete-time case, as well as additional stable zeros resulted from the column compression of $\widetilde{G}_{w}(\lambda)$. The $r_{w} \times m_{w}$ TFM of the inner factor is proper and stable and has a standard state space realization the form

$$
G_{w i}(\lambda)=\left[\begin{array}{c|c}
A_{i}-\lambda I & B_{w i} \\
\hline C_{i} & D_{w i}
\end{array}\right]
$$

Step 3. We have to compute the updated filter

as well as

$$
R_{2}(\lambda)=G_{w o}^{-1}(\lambda) R_{1}(\lambda)
$$

$$
N_{f}(\lambda)=G_{w o}^{-1}(\lambda) \widetilde{G}_{f}(\lambda)
$$


Instead of forming explicitly the realization of the inverse $G_{w o}^{-1}(\lambda)$ and then computing the minimal realizations of the above products, we can solve equivalently the linear rational system of equations

$$
G_{w o}(\lambda)\left[R_{2}(\lambda) N_{f}(\lambda)\right]=\left[R_{1}(\lambda) \widetilde{G}_{f}(\lambda)\right]
$$

Observe that $G_{w o}(\lambda), R_{1}(\lambda)$ and $\widetilde{G}_{f}(\lambda)$ have descriptor realizations which share the same state, descriptor and output matrices. Using these state space realizations, the linear rational equation (15) can be equivalently solved (see Varga [2004]) by computing first the solution $X(\lambda)$ of

$$
\left[\begin{array}{cc}
\widetilde{A}-\lambda \widetilde{E} & \bar{B}_{w} \\
\widetilde{C} & \bar{D}_{w}
\end{array}\right] X(\lambda)=\left[\begin{array}{ccc}
\widetilde{B}_{y} & \widetilde{B}_{u} & \widetilde{B}_{f} \\
\widetilde{D}_{y} & \widetilde{D}_{u} & \widetilde{D}_{f}
\end{array}\right]
$$

and then taking

With the pole pencil

$$
\left[R_{2}(\lambda) N_{f}(\lambda)\right]=\left[\begin{array}{ll}
0 & I_{q_{1}}
\end{array}\right] X(\lambda)
$$

$$
A_{w o}-\lambda E_{w o}:=\left[\begin{array}{cc}
\widetilde{A}-\lambda \widetilde{E} & \bar{B}_{w} \\
\widetilde{C} & \bar{D}_{w}
\end{array}\right]
$$

and output matrix $C_{w o}=\left[0-I_{q_{1}}\right]$ we have

$\left[R_{2}(\lambda) \mid N_{f}(\lambda)\right]=C_{w o}\left(\lambda E_{w o}-A_{w o}\right)^{-1}\left[\widehat{B}_{y} \widehat{B}_{u} \mid \widehat{B}_{f}\right]$ where

$$
\left[\begin{array}{ll}
\widehat{B}_{y} & \widehat{B}_{u} \mid \widehat{B}_{f}
\end{array}\right]:=\left[\begin{array}{cc|c}
\widetilde{B}_{y} & \widetilde{B}_{u} & \widetilde{B}_{f} \\
\widetilde{D}_{y} & \widetilde{D}_{u} & \widetilde{D}_{f}
\end{array}\right]
$$

Explicit standard realizations can be easily computed if $\bar{D}_{w}$ is invertible (i.e., $\widetilde{G}_{w}(\lambda)$ has no zeros at infinity).

Step 4. For the computation of the proper and stable coprime factorization (11), we can use an output injection matrix $L$ such that $A_{w o}+L C_{w o}-\lambda E_{w o}$ has only simple eigenvalues at infinity and all finite eigenvalues are stable. The computation of such an $L$ can be done using pole assignment algorithms for descriptor systems [Varga, 1995]. With such a choice, the factors in (11) have the realizations

$$
\begin{aligned}
{\left[R_{3}(\lambda)\right.} & \left.\widetilde{N}_{f}(\lambda)\right]=\left[\begin{array}{c|cc:c}
A_{w o}+L C_{w o}-\lambda E_{w o} & \widehat{B}_{y} & \widehat{B}_{u} & \widehat{B}_{f} \\
\hline C_{w o} & 0 & 0 & 0
\end{array}\right] \\
M_{w}(\lambda) & =\left[\begin{array}{c|c}
A_{w o}+L C_{w o}-\lambda E_{w o} & L \\
\hline C_{w o} & I_{q_{1}}
\end{array}\right]
\end{aligned}
$$

We can alternatively determine $L$ such that $A_{w o}-\lambda\left(E_{w o}-\right.$ $L C_{w o}$ ) has only simple eigenvalues at infinity and all finite eigenvalues are stable. This could be advantageous especially if only the infinite poles have to be moved to stable positions. Taking into account the particular form of the realization (17), we obtain the following realizations of the factors (see [Varga, 1998])

$$
\begin{aligned}
& {\left[R_{3}(\lambda): \tilde{N}_{f}(\lambda)\right]=\left[\begin{array}{c|c:c}
A_{w o}-\lambda\left(E_{w o}-L C_{w o}\right) & \widehat{B}_{y} \widehat{B}_{u}: \widehat{B}_{f} \\
\hline C_{w o} & 0 & 0
\end{array}\right]} \\
& M_{w}(\lambda)=\left[\begin{array}{c|c}
A_{w o}-\lambda\left(E_{w o}-L C_{w o}\right) & \lambda L \\
\hline C_{w o} & I_{q_{1}}
\end{array}\right]
\end{aligned}
$$

We sketch now the computation of $L$. Assume that $Q$ and $Z$ are orthogonal matrices which achieves the following spectral separation of the regular pencil $A_{w o}-\lambda E_{w o}$

$$
Q\left(A_{w o}-\lambda E_{w o}\right) Z=\left[\begin{array}{cc}
A_{\bar{s}}-\lambda E_{\bar{s}} & * \\
0 & A_{s}-\lambda E_{s}
\end{array}\right],
$$

where $A_{\bar{s}}-\lambda E_{\bar{s}}$ contains all unstable eigenvalues, while $A_{s}-\lambda E_{s}$ contains the stable ones. For example, in the continuous-time case, $A_{\bar{s}}-\lambda E_{\bar{s}}$ contains all eigenvalues on the imaginary axis including the infinite ones. In the discrete-time case, $A_{\bar{s}}-\lambda E_{\bar{s}}$ contains all eigenvalues on the unit circle. Let

$$
C_{w o} Z=\left[C_{\bar{s}} C_{s}\right]
$$

be partitioned according to the above eigenvalue splitting. We determine $L_{1}$ such that $A_{\bar{s}}+L_{1} C_{\bar{s}}-\lambda E_{\bar{s}}$ or $A_{\bar{s}}-\lambda\left(E_{\bar{s}}-\right.$ $L_{1} C_{\bar{s}}$ ) has only simple infinite eigenvalues and all its finite eigenvalues are stable.

Overall, the output injection matrix is computed as

$$
L=Q^{T}\left[\begin{array}{c}
L_{1} \\
0
\end{array}\right]
$$

This allows to determine an irreducible (controllable and observable) realization

or

$$
M_{w}(\lambda)=\left[\begin{array}{c|c}
A_{\bar{s}}+L_{1} C_{\bar{s}}-\lambda E_{\bar{s}} & L_{1} \\
\hline C_{\bar{s}} & I_{q_{1}}
\end{array}\right]
$$

$$
M_{w}(\lambda)=\left[\begin{array}{c|c}
A_{\bar{s}}-\lambda\left(E_{\bar{s}}-L_{1} C_{\bar{s}}\right) & \lambda L_{1} \\
\hline C_{\bar{s}} & I_{q_{1}}
\end{array}\right]
$$

A minimal realization as standard system can be easily computed by eliminating the non-dynamic modes. The McMillan order of the resulting $M_{w}(\lambda)$ is exactly the number of unstable zeros of $\widetilde{G}_{w}(\lambda)$ on the imaginary axis (including infinity) in the continuous-time case, or on the unit circle in the discrete-time case.

Step 5. In the case $\alpha=\infty$, we can choose $\Psi(\lambda)=$ $\Psi_{0}$, a $q \times q_{1}$ full row rank constant matrix, such that $\left\|\Psi_{0} M_{w}(\lambda)\right\|_{\infty}=\gamma$.

In the case $\alpha=2$, we can choose $\Psi(s)$ in the continuoustime case such that $\Psi(s) M_{w}(s)$ is strictly proper and $\left\|\Psi(s) M_{w}(s)\right\|_{2}=\gamma$. This can be easily achieved by choosing

$$
\Psi(s)=\frac{1}{s+a} \Psi_{0}
$$

where the full rank matrix $\Psi_{0}$ is chosen such that

$$
\left\|\Psi(s) M_{w}(s)\right\|_{2}=\gamma
$$

In the discrete-time case, the choice $\Psi(z)=\Psi_{0}$, with $\Psi_{0}$ a $q \times q_{1}$ full row rank constant matrix, is possible.

\section{ILLUSTRATIVE EXAMPLE}

We consider the robust fault detection example of [Edelmayer and Bokor, 2002]. The fault system (1) has a standard state space realization (13) with $E=I$ and

$$
\begin{gathered}
A\left(\delta_{1}, \delta_{2}\right)=\left[\begin{array}{ccc}
-0.8 & 0 & 0 \\
0 & -0.5\left(1+\delta_{1}\right) & 0.6\left(1+\delta_{2}\right) \\
0 & -0.6\left(1+\delta_{2}\right) & -0.5\left(1+\delta_{1}\right)
\end{array}\right] \\
B_{u}=\left[\begin{array}{ll}
1 & 1 \\
1 & 0 \\
0 & 1
\end{array}\right], B_{d}=0, B_{f}=\left[\begin{array}{ll}
1 & 1 \\
1 & 0 \\
0 & 1
\end{array}\right], C=\left[\begin{array}{lll}
0 & 1 & 1 \\
1 & 1 & 0
\end{array}\right] \\
D_{u}=0, D_{d}=0, D_{f}=0 .
\end{gathered}
$$

In the expression of $A\left(\delta_{1}, \delta_{2}\right), \delta_{1}$ and $\delta_{2}$ are uncertainties in the real and imaginary parts of the two complex conjugated eigenvalues $\lambda_{1,2}=-0.5 \pm j 0.6$ of the nominal value $A(0,0)$. The fault detector filter is aimed to provide robust fault detection of actuator faults in the presence of these parametric uncertainties. 
We reformulate the problem by assimilating $\delta_{1}$ and $\delta_{2}$ with fictitious noise inputs. We take $A$ in (13) simply as the nominal value $A(0,0)$ and additionally define

$$
B_{w}=\left[\begin{array}{ll}
0 & 0 \\
0 & 1 \\
1 & 0
\end{array}\right], \quad D_{w}=0
$$

We wish to design a residual generator to detect actuator faults, which provides the largest possible gap $\beta / \gamma$. For this we employ a detector with two outputs (i.e., we choose $q=2)$.

At Step 1 we can choose as initial detector

$$
R_{1}(\lambda)=\left[I_{2}-G_{u}(s)\right]=\left[\begin{array}{c|cc}
A-s I & 0-B_{u} \\
\hline C & I-D_{u}
\end{array}\right]
$$

$G_{w}(s)$ is invertible and has three zeros at $\{-0.8, \infty, \infty\}$. The finite zero -0.8 is in fact a non-controllable eigenvalue of $A$ for the pair $\left(A, B_{w}\right)$. No inner-outer factorization is necessary and we simply set at Step $2 G_{w o}(s)=G_{w}(s)$, and $G_{w i}(s)=I$.

At Step 3 we can immediately build the realizations of $R_{2}(s)$ and $N_{f}(s)$, with

$$
A_{w o}-s E_{w o}=\left[\begin{array}{cc}
A-s I & B_{w} \\
C & D_{w}
\end{array}\right], \quad C_{w o}=\left[\begin{array}{ll}
0 & -I_{2}
\end{array}\right]
$$

To compute the proper and stable factorization at Step 4 we use the orthogonal (permutation) matrices

$$
Q=\left[\begin{array}{lllll}
0 & 1 & 0 & 0 & 0 \\
0 & 0 & 1 & 0 & 0 \\
0 & 0 & 0 & 1 & 0 \\
0 & 0 & 0 & 0 & 1 \\
1 & 0 & 0 & 0 & 0
\end{array}\right], \quad Z=\left[\begin{array}{lllll}
0 & 0 & 0 & 0 & 1 \\
0 & 0 & 1 & 0 & 0 \\
0 & 0 & 0 & 1 & 0 \\
0 & 1 & 0 & 0 & 0 \\
1 & 0 & 0 & 0 & 0
\end{array}\right]
$$

to put the pencil $A_{w_{o}}-s E_{w o}$ into an infinite-finite separated form $\widetilde{A}_{w o}-s \widetilde{E}_{w o}:=Q A_{w o} Z-s Q E_{w o} Z$ such that

$$
\begin{gathered}
\widetilde{A}_{w o}-s \widetilde{E}_{w o}=\left[\begin{array}{rrrr:rl}
1 & 0 & -0.5-s & 0.6 & 0 \\
0 & 1 & -0.6 & -0.5-s & 0 \\
0 & 0 & 1 & 1 & 0 & 0 \\
0 & 0 & 1 & 0 & 1 \\
\hdashline 0 & 0 & 0 & 0 & -0.8-s
\end{array}\right] \\
Q\left[\begin{array}{rr|r}
0 & -B_{u} & B_{f} \\
I_{p}-D_{u} & D_{f}
\end{array}\right]=\left[\begin{array}{rrrr|rr}
0 & 0 & -1 & 0 & 1 & 0 \\
0 & 0 & 0 & -1 & 0 & 1 \\
1 & 0 & 0 & 0 & 0 & 0 \\
0 & 1 & 0 & 0 & 0 & 0 \\
\hdashline 0 & 0 & -1 & -1 & 1 & 1
\end{array}\right] \\
\widetilde{C}_{w o}:=C_{w o} Z=\left[\begin{array}{rrrr:r}
0 & -1 & 0 & 0 & 0 \\
-1 & 0 & 0 & 0 & 0
\end{array}\right]
\end{gathered}
$$

If we choose

$$
L=\left[\begin{array}{c}
\widetilde{L}_{1} \\
0
\end{array}\right]=\left[\begin{array}{rr}
0 & -1 \\
-1 & 0 \\
0 & 0 \\
0 & 0 \\
\hdashline 0 & 0
\end{array}\right]
$$

the generalized eigenvalues of the pair $\left(\widetilde{A}_{w o}, \widetilde{E}_{w o}-L \widetilde{C}_{w o}\right.$ are $\{\infty, \infty,-1,-1,-0.8\}$ and the infinite eigenvalues arı simple. The realization of the proper denominator facto $M_{w}(\lambda)$ is constructed according to (19)

$$
M_{w}(s)=\left[\begin{array}{cc|cc}
1+s & 0 & 0 & -s \\
0 & 1+s & -s & 0 \\
\hline 0 & -1 & 1 & 0 \\
-1 & 0 & 0 & 1
\end{array}\right]=\left[\begin{array}{cc}
\frac{1}{s+1} & 0 \\
0 & \frac{1}{s+1}
\end{array}\right]
$$

The realization of $R_{3}(s)$ and $\widetilde{N}_{f}(s)$ are computed according to (18). With $\Psi(s)=I$, the resulting detector is $R(s)=R_{3}(s)$ and has a standard state-space realization

$$
R(s)=\left[\begin{array}{c|cc}
A_{R}-s I & B_{R_{y}} & B_{R_{u}} \\
\hline C_{R} & D_{R_{y}} & D_{R_{u}}
\end{array}\right],
$$

where

$$
\begin{gathered}
A_{R}=\left[\begin{array}{rrc}
-1 & 0 & 0.07071 \\
0 & -1 & 0.7778 \\
0 & 0 & -0.8
\end{array}\right], \quad C_{R}=\left[\begin{array}{ccr}
0 & 1.414 & -1 \\
1.414 & 0 & 1
\end{array}\right] \\
{\left[B_{R_{y}} \mid B_{R_{u}}\right]=\left[\begin{array}{cc|cc}
-0.4243 & 0.0707 & -0.7071 & 0 \\
-0.3536 & 0.7778 & 0 & -0.7071 \\
0 & 0 & -1 & -1
\end{array}\right]} \\
{\left[D_{R_{y}} \mid D_{R_{u}}\right]=\left[\begin{array}{lr|rr}
1 & -1 & 0 & 0 \\
0 & 1 & 0 & 0
\end{array}\right]}
\end{gathered}
$$

The resulting $R_{f}(s)=\widetilde{N}_{f}(s)$ has the standard realization

$$
R_{f}(s)=\left[\begin{array}{c|c}
A_{R}-s I & B_{R_{f}} \\
\hline C_{R} & 0
\end{array}\right]
$$

where

$$
B_{R_{f}}=\left[\begin{array}{cc}
0.7071 & 0 \\
0 & 0.7071 \\
1 & 1
\end{array}\right]
$$

The computed detector $R(s)$ ensures that for $\gamma=1$, we obtain $\beta=\left\|\widetilde{N}_{f}(s)\right\|_{-}=1.7766$.

The same performance can be achieved also with a scalar output detector $(q=1)$, by choosing $\Psi_{0}=$ $\left[\begin{array}{ll}0.6377 & 0.7702\end{array}\right]$. In this case, the optimal scalar output detector $R(s)$ has a second order minimal state space realization.

In Figure 1 we present the results of a Monte Carlo analysis of step responses from control inputs and from the fault inputs for 100 random samples of $\delta_{1}$ and $\delta_{2}$ in the range $[-0.5,0.5]$. As can be observed, with an appropriate choice of the detection threshold, the detection of constant faults can be reliably performed in the presence of parametric uncertainties.

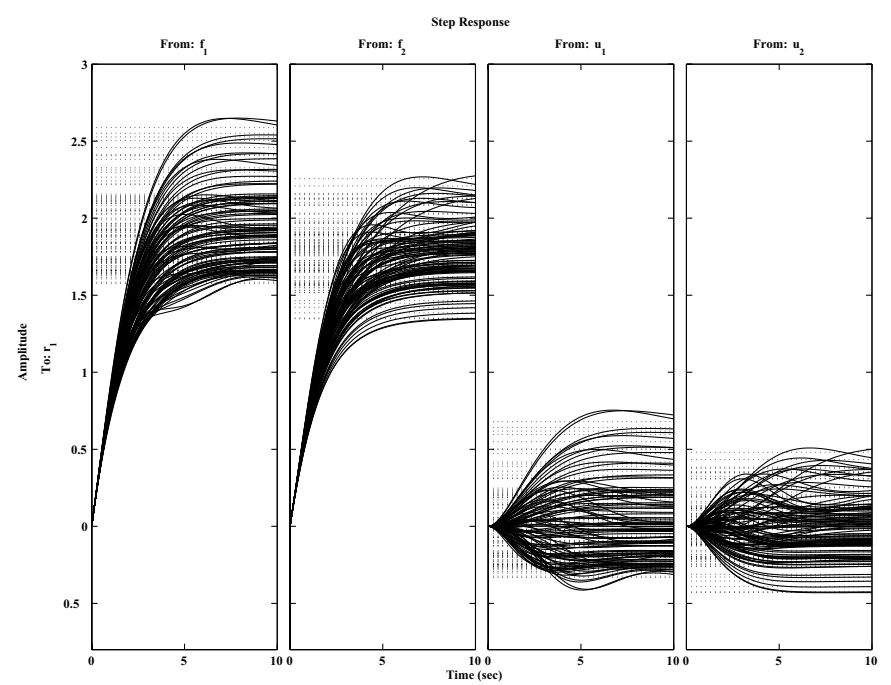

Fig. 1. Monte Carlo step response analysis of detector robustness for uncertainties in the real and imaginary parts of the complex conjugated eigenvalues 


\section{CONCLUSIONS}

In this paper we developed the AFDPO Procedure, which represents a general computational approach to solve optimal fault detection problems. An important feature of this procedure is that it allows to compute an exact solution of the AFDP whenever such a solution exists. This contrasts with some existing procedures [Jaimoukha et al. 2006, Liu and Zhou, 2007a], where the existence of an exact solution is blurred into the problem formulation, and even if an exact solution exists (see the used illustrative examples) this can not be computed. The $\mathcal{H}_{-} / \mathcal{H}_{2}$ solution of the AFDP can be also seen as an enhancement of the solution of the stochastic fault detection problem handled in [Nikoukhah, 1994]. The AFDPO Procedure can be used with $q=1$ to design a bank of detectors for structured residuals with a desired fault signature.

We also discussed in details the computational ingredients necessary to perform the AFDPO Procedure using state space techniques. The state space version of this new procedure is an example of an integrated algorithm, where instead of using standard computational modules to perform each computational step independently, the successive computational steps are linked via the resulting data structures at the end of each processing step. This allows to exploit the intrinsic structural features of all subproblems to be solved and leads to highly efficient computations. A key aspect for such an approach is the usage of descriptor system representations, which allows to handle both initial and intermediary quantities without special care for properness.

An important computational ingredient for the proposed approach is an inner-outer factorization algorithm [Oară and Varga, 2000, 1999]. This algorithm is generally applicable to both standard or descriptor systems, regardless if zeros are present on the imaginary axis (including infinity) in the continuous-time case or on the unit circle in the discrete-time case. If such zeros are present, they are automatically included in the outer factor. A nice feature exploited in the proposed procedure is that the resulting quasi-outer factor preserves the state and output matrices of the original realization.

The final adjustment of detector poles is usually necessary to enforce a desired dynamics of the detection system. This could be important, since otherwise the resulting dynamics is partly determined by the zeros of the outer factor, and may not be sufficiently rapid to guarantee fast detections. This aspect as well as the optimal setting of the free parameters at the last step still require a closer examination.

For all computations numerically reliable algorithms are available and are implemented in the DESCRIPTOR SYSTEMS Toolbox developed since 2000 by the author [Varga, 2000]. An efficient implementation of the AFDPO Procedure has been recently included in the FAULT DETECTION Toolbox [Varga, 2006].

\section{REFERENCES}

J. Chen and R. J. Patton. Robust Model-Based Fault Diagnosis for Dynamic Systems. Kluwer Academic Publishers, London, 1999.

S. X. Ding, P. M. Frank, E. L. Ding, and T. Jeinsch. A unified approach to the optimization of fault detection systems. Int. J. of Adaptive Contr. and Signal Processing, 14:725-745, 2000.

A. Edelmayer and J. Bokor. Optimal $\mathcal{H}_{\infty}$ scaling for sensitivity optimization of detection filters. Int. J. Robust Nonlinear Control, 12:749-760, 2002.

J. Gertler. Fault Detection and Diagnosis in Engineering Systems. Marcel Dekker, New York, 1998.

I. M. Jaimoukha, Z. Li, and V. Papakos. A matrix factorization solution to the $\mathcal{H}_{-} / \mathcal{H}_{\infty}$ fault detection problem. Automatica, 42:1907-1912, 2006.

N. Liu and K. Zhou. Optimal solutions to multiobjective robust fault detection problems. Proc. of $C D C^{\prime} 0^{7} 7, \mathrm{New}$ Orleans, USA, 2007a.

N. Liu and K. Zhou. Optimal robust fault detection for linear discrete time systems. Proc. of $C D C^{\prime} 0^{7}$, New Orleans, USA, 2007b.

R. Nikoukhah. Innovations generation in the presence of unknown inputs: application to robust failure detection. Automatica, 30:1851-1867, 1994.

C. Oară and A. Varga. Computation of general inner-outer and spectral factorizations. IEEE Trans. Automat. Control, 45:2307-2325, 2000.

C. Oară and A. Varga. The general inner-outer factorization problem for discrete-time systems. Proc. ECC'99, Karlsruhe, Germany, 1999.

A. Saberi, A. A. Stoorvogel, P. Sannuti, and H. Niemann. Fundamental problems in fault detection and identification. Int. J. Robust Nonlinear Control, 10:1209-1236, 2000.

A. Varga. A Descriptor Systems toolbox for Matlab. Proc. CACSD'2000 Symposium, Anchorage, Alaska, 2000.

A. Varga. On computing least order fault detectors using rational nullspace bases. Proc. of IFAC Symp. SAFEPROCESS'2003, Washington D.C., 2003.

A. Varga. Computation of least order solutions of linear rational equations. Proc. of MTNS'04, Leuven, Belgium, 2004.

A. Varga. A fault detection toolbox for Matlab. Proc. of CACSD'06, Munich, Germany, 2006.

A. Varga. On designing least order residual generators for fault detection and isolation. Proc. 16th Internat. Conf. on Control Systems and Computer Science, Bucharest, Romania, pages 323-330, 2007.

A. Varga. On computing nullspace bases - a fault detection perspective. Proc. IFAC 2008 World Congress, Seoul, Korea., pages 6295-6300, 2008.

A. Varga. On stabilization of descriptor systems. Systems \& Control Letters, 24:133-138, 1995.

A. Varga. Computation of coprime factorizations of rational matrices. Lin. Alg. \& Appl., 271:83-115, 1998.

P. Zhang and S. X. Ding. An integrated trade-off design of observer based fault detection systems. Automatica, 44:1886-1894, 2008. 